\title{
Study of charmoniumlike states by amplitude analyses at Belle
}

\author{
Kirill Chilikin ${ }^{1, *}$ \\ ${ }^{1}$ Lebedev Physical Institute of the Russian Academy of Sciences, Moscow
}

Abstract. A review of charmoniumlike state studies at Belle which use amplitude analyses is presented, including the $Z_{c}(4430)^{+}, Z_{c}(4200)^{+}$and $\chi_{c 0}(3860)$.

\section{Introduction}

Recently, many new states containing a $c \bar{c}$ mesons have been observed; many of them do not match the expectations for the conventional quark-antiquark charmonium states [1]. Specifically, the charged charmoniumlike states have the minimal quark content $|c \bar{c} u \bar{d}\rangle$; thus, they are necessarily exotic. This review includes the studies of charmoniumlike states that were performed by the Belle Collaboration using amplitude analyses: the measurement of the $Z_{c}(4430)^{+}$quantum numbers [2], the observation of the $Z_{c}(4200)^{+}$[3], and the observation of the $\chi_{c 0}(3860)$ [4]. The first two analyses study the charged charmoniumlike states, while the $\chi_{c 0}(3860)$ is an alternative candidate for the conventional charmonium state $\chi_{c 0}(2 P)$.

\section{Analyses}

\subsection{Measurement of the $Z_{c}(4430)^{+}$quantum numbers}

The $Z_{c}(4430)^{+}$quantum numbers were measured by performing an amplitude analysis of the decays $\bar{B}^{0} \rightarrow \psi(2 S) \pi^{+} K^{-}$[2] (inclusion of charge-conjugate modes is implied hereinafter). The Belle Collaboraton previously observed the $Z_{c}(4430)^{+}$in a one-dimensional analysis of the same decay [5] and later using a Dalitz analysis [6]. However, the previous Belle analyses either ignored the interference with the contribution of the $K^{*}$ resonances completely or reduced the sensitivity by integrating over the angular variables; consequently, they were not sensitive to the $Z_{c}(4430)^{+}$quantum numbers. Also, before the $Z_{c}(4430)^{+}$quantum-number determination, a model-independent analysis had been performed by the BaBar Collaboration [7]; the $Z_{c}(4430)^{+}$existence had not been confirmed, but the result had not contradicted the Belle observation because of a smaller data sample.

The default model included six $K^{*}$ resonances $\left(K_{0}^{*}(800), K^{*}(892), K^{*}(1410), K_{0}^{*}(1430)\right.$, $K_{2}^{*}(1430)$, and $\left.K^{*}(1680)\right)$ and the $Z_{c}(4430)^{+}$. All possible quantum numbers of the $Z_{c}(4430)^{+}$ with $J \leq 2$ were considered: $0^{-}, 1^{+}, 1^{-}, 2^{+}, 2^{-}$. The preferred $Z_{c}(4430)^{+}$spin-parity hypothesis was found to be $1^{+}$. Projections of the fit results onto $M_{\psi(2 S) \pi^{+}}^{2}$ in the models without the $Z_{c}(4430)^{+}$and with the $Z_{c}(4430)^{+}\left(J^{P}=1^{+}\right)$are shown in figure 1 . The $Z_{c}(4430)^{+}$parameters were measured to be $M=4485_{-22-11}^{+22+28} \mathrm{MeV} / c^{2}$ and $\Gamma=200_{-46-35}^{+41+26} \mathrm{MeV}$.

*e-mail: chilikin@lebedev.ru 


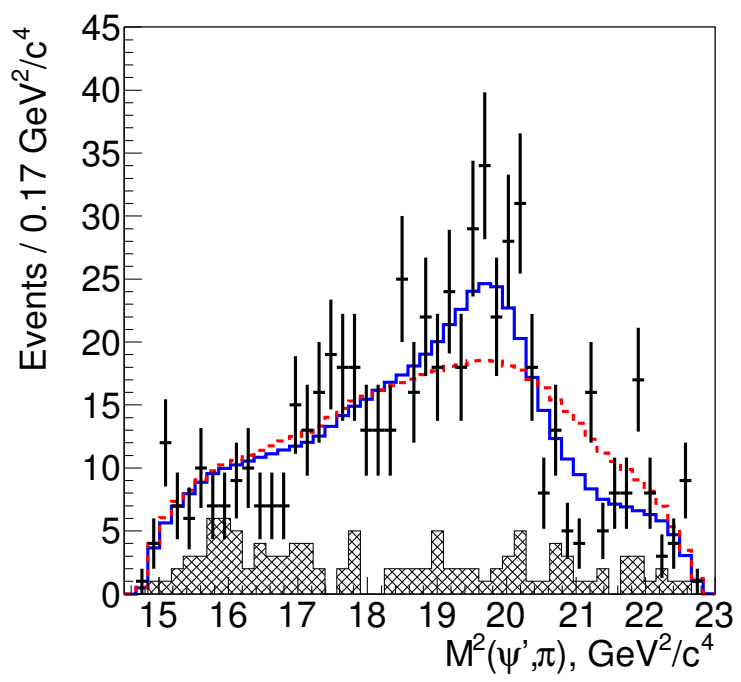

Figure 1. Projections of the $\bar{B}^{0} \rightarrow \psi(2 S) \pi^{+} K^{-}$fit results onto $M_{\psi(2 S) \pi^{+}}^{2}$. The points with error bars are the data, the hatched histogram is the background, the blue solid line is the fit with the $Z_{c}(4430)^{+}$, and the red dashed line is the fit without its contribution. The narrow $K^{*}$ veto is applied: $\left|M_{K^{-} \pi^{+}}-m_{K^{*}(892)}\right|>$ $100 \mathrm{MeV}$ and $\left|M_{K^{-} \pi^{+}}-m_{K_{2}^{*}(1430)}\right|>100 \mathrm{MeV}$, where $m_{K^{*}}$ are the nominal masses of the specified $K^{*}$ resonances. Figure from reference [2].

Comparison of the quantum-number hypotheses was performed using Monte-Carlo (MC) pseudoexperiments generated in accordance with the fit results. It was performed for various amplitude models to take the systematic uncertainty into account. The resulting exclusion levels of the $0^{-}, 1^{-}, 2^{-}$, and $2^{+}$hypotheses were found to be $3.4 \sigma, 3.7 \sigma, 4.7 \sigma$, and $5.1 \sigma$, respectively.

The observation of the $Z_{c}(4430)^{+}$and the results of the measurement its quantum numbers were later confirmed by the LHCb Collaboration [8]. The LHCb Collaboration additionally observed the resonant character of the $Z_{c}(4430)^{+}$by measuring the dependence of its amplitude on mass. The $Z_{c}(4430)^{+}$existence was also confirmed by LHCb in a model-independent way [9].

\subsection{Observation of the $Z_{c}(4200)^{+}$}

The $Z_{c}(4200)^{+}$was observed in the decays $\bar{B}^{0} \rightarrow J / \psi \pi^{+} K^{-}$[3]. The default model included $10 K^{*}$ resonances: $K_{0}^{*}(800), K^{*}(892), K^{*}(1410), K_{0}^{*}(1430), K_{2}^{*}(1430), K^{*}(1680), K_{3}^{*}(1780)$, $K_{0}^{*}(1950), K_{2}^{*}(1980), K_{4}^{*}(2045)$. The $Z_{c}(4430)^{+}$was also included to the default model. Optionally, an additional $Z_{c}^{+}$resonance was added. All possible quantum numbers of the new $Z_{c}^{+}$ state with $J \leq 2$ were considered. Its significance was calculated globally with the expected null-hypothesis distribution of $\Delta(-2 \ln L)$ determined from MC pseudoexperiments. A new charmoniumlike state $Z_{c}(4200)^{+}$with $J^{P}=1^{+}$was found with a significance of $6.2 \sigma$. Its parameters were measured to be $M=4196_{-29-13}^{+31+17} \mathrm{MeV} / c^{2}$ and $\Gamma=370_{-70-132}^{+70} \mathrm{MeV}$. Projections of the fit results onto $M_{J / \psi \pi^{+}}$in the models with and without the $Z_{c}(4200)^{+}$are shown in figure 2 . 


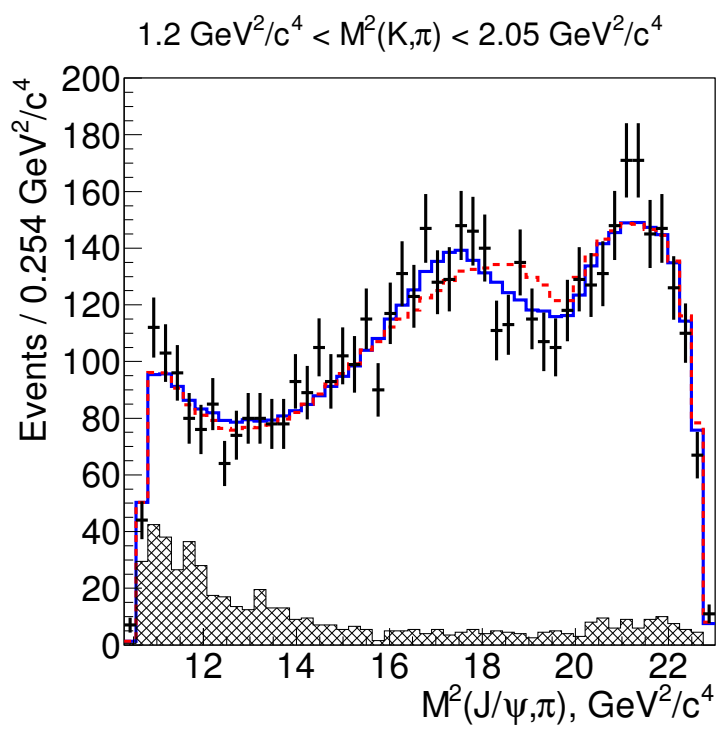

Figure 2. Projections of the $\bar{B}^{0} \rightarrow J / \psi \pi^{+} K^{-}$fit results onto $M_{J / \psi \pi^{+}}^{2}$. The points with error bars are the data, the hatched histogram is the background, the blue solid line is the fit with the $Z_{c}(4200)^{+}$, and the red dashed line is the fit without its contribution. The region with $1.2<M_{K^{-} \pi^{+}}^{2}<2.05 \mathrm{GeV} / c^{2}$ is selected. Figure from reference [3].

The comparison of the quantum numbers was performed by using $\Delta(-2 \ln L)$; it was checked using the pseudoexperiment method. The $0^{-}, 1^{-}, 2^{-}$, and $2^{+}$hypothesis were found to be excluded at the levels of $6.1 \sigma, 7.4 \sigma, 4.4 \sigma$, and $7.0 \sigma$, respectively.

In addition, the mass dependence of the $Z_{c}(4200)^{+}$amplitude was measured by performing an alternative fit with the $Z_{c}(4200)^{+}$contribution represented by complex constants in six mass bins. The resulting Argand plot for the $H_{1}$ amplitude shows a resonant dependence; it is presented in figure 3 . The $H_{0}$ amplitudes have much larger relative errors and it is not possible to determine whether they also change resonantly.

The significance of the $Z_{c}(4430)^{+}$was measured to be $4.0 \sigma$. Thus, evidence for $Z_{c}(4430)^{+} \rightarrow J / \psi \pi^{+}$was found. The $Z_{c}(4430)^{+}$interferes destructively with the $K^{*}$ resonances.

The presence of exotic $J / \psi \pi^{+}$contribution in the decays $\bar{B}^{0} \rightarrow J / \psi \pi^{+} K^{-}$was recently confirmed by the LHCb Collaboration [10]. The observed distribution of the $J / \psi \pi^{+}$invariant mass suggests the existence of two resonances with masses of two resonances with $M_{J / \psi \pi^{+}} \approx$ $4200 \mathrm{MeV} / c^{2}$ and $M_{J / \psi \pi^{+}} \approx 4600 \mathrm{MeV} / c^{2}$. The analysis performed by LHCb is modelindependent and it consequently does not measure the masses, widths, and quantum numbers of the $J / \psi \pi^{+}$resonances. In addition, the $Z_{c}(4200)^{+}$state may be the same as the $Z_{c}(4240)^{+}$ (or $R_{c 0}(4240)^{+}$) state found by LHCb in the decays $\bar{B}^{0} \rightarrow \psi(2 S) \pi^{+} K^{-}$[8] if the $Z_{c}(4240)^{+}$ quantum numbers are $J^{P}=1^{+}$(the preferred hypothesis is $0^{-}$, but it is preferred over $1^{+}$at $1 \sigma$ level only).

\subsection{Observation of an alternative $\chi_{c 0}(2 P)$ candidate}

The $\chi_{c 0}(3860)$ was observed in the process $e^{+} e^{-} \rightarrow J / \psi D \bar{D}$ [4], where $D$ means either $D^{0}$ or $D^{+}$. The analysis was motivated by the inconsistency of the observed properties 


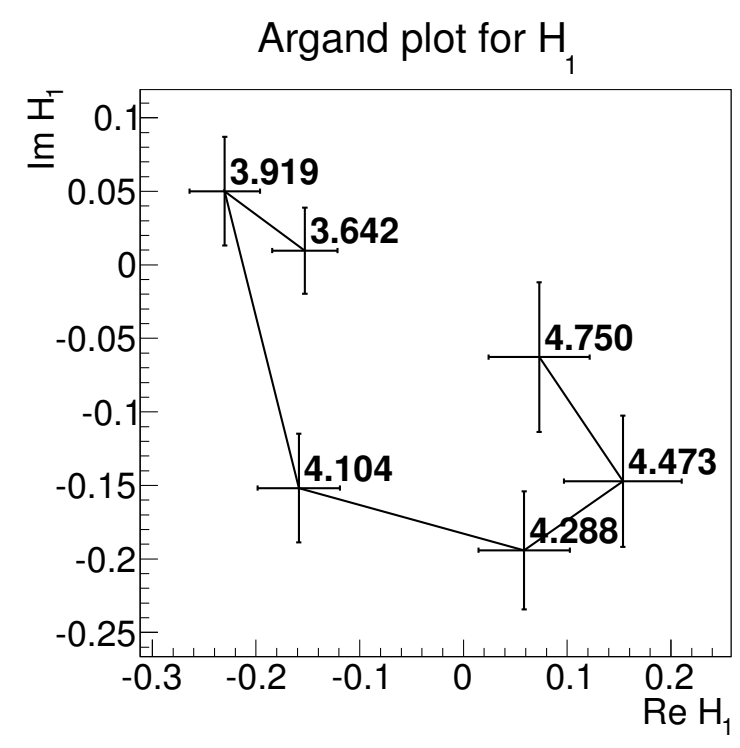

Figure 3. Argand plot for the $Z_{c}(4200)^{+}$helicity amplitude $H_{1}$. The bin central mass values (in $\mathrm{GeV} / c^{2}$ ) are shown near the points. Figure from reference [3].

of the $X(3915)$ and those expected for the $\chi_{c 0}(2 P)$ state $[11,12]$. The Belle Collaboration had previously observed new charmoniumlike states $X(3940)$ and $X(4160)$ in the processes $e^{+} e^{-} \rightarrow J / \psi D^{(*)} \bar{D}^{(*)}[13]$; the process $e^{+} e^{-} \rightarrow J / \psi \chi_{c 0}(1 P)$ had also been observed by Belle $[14,15]$ before the $\chi_{c 0}(2 P)$ search.

Since the signal yield in the processes $e^{+} e^{-} \rightarrow J / \psi D^{(*)} \bar{D}^{(*)}$ is known to be rather low even with a partial reconstruction, which includes only the $J / \psi$ and one of the $D^{(*)}$ mesons, the event selection was optimized to improve the sensitivity. A multivariate analysis was performed for each $D$ decay channel. After that, a global optimization of the selection requirements has been performed, which included the channel-specific definitions of the signal region and the neural-network output requirements.

The default model of the process $e^{+} e^{-} \rightarrow J / \psi D \bar{D}$ included the nonresonant amplitude (with three different parameterizations) and an additional resonance $X^{*}$ decaying to $D \bar{D}$. The $X^{*}$ produced in this process has even spin and positive parity; the $C$-parity should also be positive. Thus, two quantum-number hypotheses were considered: $J^{P C}=0^{++}$and $2^{++}$. A new charmoniumlike state $X^{*}(3860)$ was observed with a significance of $6.5 \sigma$. The preferred quantum-number hypothesis is $J^{P C}=0^{++}$(thus, the new state can be denoted alternatively as the $\chi_{c 0}(3860)$ [16]), however, the $2^{++}$hypothesis was not excluded: the $0^{++}$hypothesis is preferred at $2.5 \sigma$ level. The $\chi_{c 0}(3860)$ parameters were measured to be $M=3862_{-32-13}^{+26+40} \mathrm{MeV} / c^{2}$ and $\Gamma=201_{-67-82}^{+154+88} \mathrm{MeV}$. Projections of the fit results onto $M_{D \bar{D}}$ in the models with and without the $\chi_{c 0}(3860)$ are shown in figure 2.

The new $\chi_{c 0}(3860)$ resonance seems to be a better candidate for the $\chi_{c 0}(2 P)$ charmonium state than the $X(3915)$, because its properties are well matched to expectations for the $\chi_{c 0}(2 P)$ resonance. Such properties include the mass, the mass difference with the $\chi_{c 2}(2 P)$, the largewidth $D \bar{D}$ observation decay mode, the production that is consistent with pure $S$-wave as is the case for the process $e^{+} e^{-} \rightarrow J / \psi \chi_{c 0}(1 P)$. In addition, the $\chi_{c 0}(3860)$ mass and width 


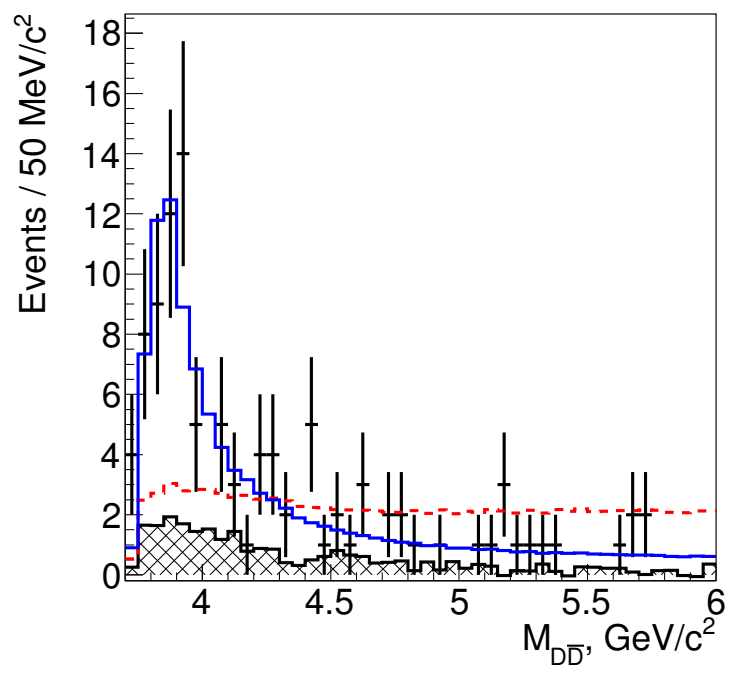

Figure 4. Projections of the $e^{+} e^{-} \rightarrow J / \psi D^{(*)} \bar{D}^{(*)}$ fit results onto $M_{D \bar{D}}$. The points with error bars are the data, the hatched histogram is the background, the blue solid line is the fit with the $X^{*}$ resonance $\left(J^{P C}=\right.$ $\mathrm{O}^{++}$), and the red dashed line is the fit with nonresonant amplitude only. Figure from reference [4].

agree with the $\chi_{c 0}(2 P)$ parameters determined from an alternative fit to the Belle and BaBar $\gamma \gamma \rightarrow D \bar{D}$ data $[11]$.

\section{References}

[1] S. L. Olsen, T. Skwarnicki and D. Zieminska, Rev. Mod. Phys. 90, 015003 (2018).

[2] K. Chilikin et al. (Belle Collaboration), Phys. Rev. D 88, 074026 (2013).

[3] K. Chilikin et al. (Belle Collaboration), Phys. Rev. D 90, 112009 (2014).

[4] K. Chilikin et al. (Belle Collaboration), Phys. Rev. D 95, 112003 (2017).

[5] S. K. Choi et al. (Belle Collaboration), Phys. Rev. Lett. 100, 142001 (2008).

[6] R. Mizuk et al. (Belle Collaboration), Phys. Rev. D 80, 031104 (2009).

[7] B. Aubert et al. (BaBar Collaboration), Phys. Rev. D 79, 112001 (2009).

[8] R. Aaij et al. (LHCb Collaboration), Phys. Rev. Lett. 112, 222002 (2014).

[9] R. Aaij et al. (LHCb Collaboration), Phys. Rev. D 92, 112009 (2015).

[10] R. Aaij et al. (LHCb Collaboration), arXiv:1901.05745 [hep-ex].

[11] F. K. Guo and U. G. Meissner, Phys. Rev. D 86, 091501 (2012).

[12] S. L. Olsen, Phys. Rev. D 91, 057501 (2015).

[13] P. Pakhlov et al. (Belle Collaboration), Phys. Rev. Lett. 100, 202001 (2008).

[14] K. Abe et al. (Belle Collaboration), Phys. Rev. D 70, 071102 (2004).

[15] K. Abe et al. (Belle Collaboration), Phys. Rev. Lett. 98, 082001 (2007).

[16] M. Tanabashi et al. (Particle Data Group), Phys. Rev. D 98, 030001 (2018). 